(NASA-CR-200035) A MODIFIED

N96-17182

THERMAL CONDUCTIVITY FOR LOW

DENSITY PLASMA MAGNETIC FLUX TUBES

(NASA. Marshall Space Flight

Unclas

(enter) $4 \mathrm{p}$

G3/75 0092359 


\title{
A modified thermal conductivity for low density plasma magnetic flux tubes
}

\author{
R. H. Comfort ${ }^{1}$, P. D. Craven ${ }^{2}$, and P. G. Richards ${ }^{1}$
}

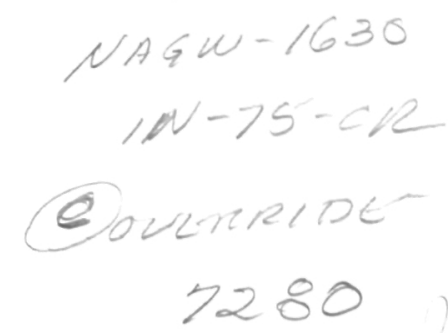

\begin{abstract}
In response to inconsistencies which have arisen in results from a hydrodynamic model in simulations of high ion temperatures (1-2 eV) observed in low density, outer plasmasphere flux tubes, we postulate a reduced thermal conductivity coefficient in which only particles in the loss cone of the quasicollisionless plasma contribute to the thermal conduction. Other particles are assumed to magnetically mirror before they reach the topside ionosphere and therefore not to remove thermal energy from the plasmasphere. This concept is used to formulate a mathematically simple, but physically limiting model for a modified thermal conductivity coefficient. When this modified coefficient is employed in the hydrodynamic model in a case study, the inconsistencies between simulation results and observations are largely resolved. The high simulated ion temperatures are achieved with significantly less heat input, and result in substantially lower ion temperatures in the topside ionosphere. We suggest that this mechanism may be operative under the limited low density, refilling conditions in which high ion temperatures are observed.
\end{abstract}

\section{Introduction}

In a recent case study examining the nature of heating mechanisms required to produce high ion temperatures frequently observed in low density plasma flux tubes at high altitudes near the plasmapause, Comfort et al. [1995] found that that these temperatures could be readily achieved, provided the heating mechanism operated directly on the ions (as described below), rather than through the thermal electrons. However, the resulting simulations produced some effects which are not observed. In particular, ion temperatures in the topside ionosphere are up to $5000 \mathrm{~K}$ higher than observed, and heavy ion $\left(\mathrm{O}^{+}\right)$concentrations at high altitudes are also much higher than observed. While these results are extreme examples, similar problems have been found in other case studies, e.g. Horwitz et al. [1990], Craven et al. [1995]. Comfort et al. [1995] suggest that these inconsistencies between model results and observations could be diminished if the thermal conductivity were not so large, so that less thermal energy is transported to the topside ionosphere, where $\mathrm{O}^{+}$is the dominant ion.

In the early years of in situ observations, modifications to the electron thermal conductivity were suggested to explain the high electron temperatures observed [Mayr and Volland, 1967; Mayr et al., 1973]. These modifications were based on the variability of the mean free path over it's range, and a non-linear

${ }^{1}$ Center for Space Plasma and Aeronomic Research

University of Alabama in Huntsville,Huntsville, AL 35899

${ }^{2}$ Space Sciences Laboratory, NASA/Marshall Space Flight Center Huntsville, AL 35816

Copyright 1995 by the American Geophysical Union.

Paper number 95GL02408

0094-8534/95/95GL-02408\$03.00 variation of electron temperature over this range. Predicated on the argument that much more thermal energy was transported by electrons than by ions, the ion thermal conductivity was not treated explicitly. More recently, as numerical simulations have become more sophisticated and more numerous, particularly for high latitudes, thermal conductivities have again been subjected to modification, frequently associated as much with the simulation conditions as with the plasma physical conditions [e.g. Mitchell et al., 1992].

The purpose of this report is to propose and test a mechanism which would decrease ion thermal conduction for a limited range of physical conditions. This should increase plasmaspheric ion temperatures with less heat input in the low density plasma flux tubes where high ion temperatures are most frequently observed [Comfort et al., 1988]. In the next section we describe the physical origin of the proposed reduction in thermal conductivity and then use this as a basis for formulating a simple mathematical model. In the following section, this model is applied in a simulation and results with and without the modified thermal conductivity are compared. These results and possible refinements are then discussed and conclusions are summarized.

\section{Physical Mechanism and Mathematical Model}

The basic idea is most easily understood in the context of a mean free path formulation of thermal conductivity. The modification proposed can then be applied to the more complete and accurate expression derived from kinetic theory. In the mean free path approach, information regarding the thermal properties of a fluid for a given location is carried to another location over distances of the order of a mean free path (e.g., see derivations in Chapman and Cowling [1970, Chapter 6]). These mean free paths are limited by particle collisions; in the plasmasphere, these are ion-ion (Coulomb) collisions. If densities are sufficiently low that collisions are rare, the particle motion will be essentially collisionless, and the magnetic moment will be conserved.

Suppose that the free path is quite long, on the order of the length of a flux tube or greater. Those particles which are in the atmospheric loss cone will travel to the topside ionosphere and lose their energy in collisions with ambient particles. However, the many particles which are not in the loss cone will be magnetically mirrored before they undergo a collision. Consequently, they are returned through the region which they have just passed without collision and may well suffer no collision on the return trip. As a result, the energy carried away from the location of interest is returned to that location; and, to the extent that it was included in the heat to be deposited in other locations, the thermal conductivity is in error. The proposal here is straightforward: we suggest that only those particles which are in the loss cone contribute to the thermal conductivity. This is indicated schematically in Figure 1. While this situation does not apply generally, there will be some threshold density, below which it will. For the present, we assume that it holds and investigate the consequences. 


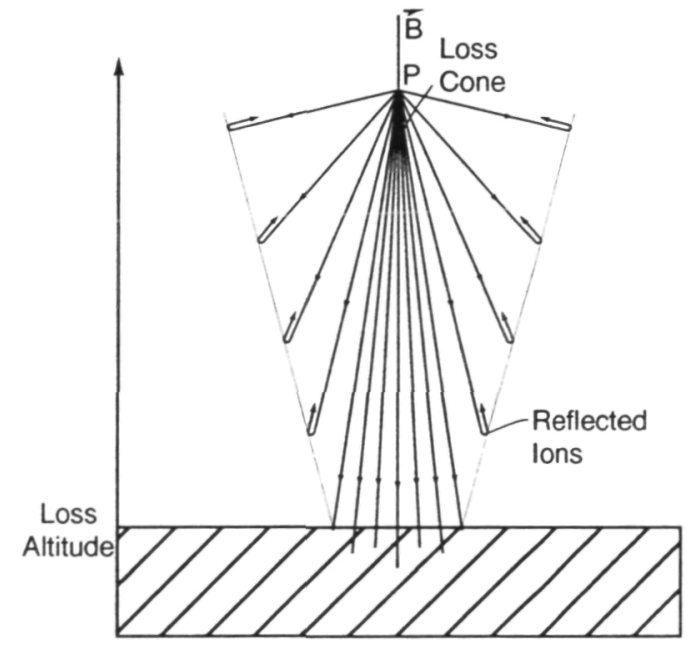

Figure 1. Schematic representation of thermal conduction limited to the atmospheric loss cone. Ions which are reflected are assumed to transport no heat from the plasmasphere.

In order to formulate a mathematically simple model, we presume that densities are sufficiently low, that ions are essentially collisionless until they descend to some loss altitude $r_{L}$, below which they are assumed lost through collisions. The magnitude of the magnetic field at that altitude, $B_{L}$, defines the loss cone of the particle distribution at any higher location through the conservation of the magnetic moment. That is, particles whose mirror field satisfies the relation $B_{m} \geq B_{L}$ will be lost. Correspondingly, particles with pitch angles satisfying $\alpha \leq$ $\alpha_{L}$ will be lost; and only these particles will contribute to the thermal conductivity. We assume an isotropic Maxwellian distribution (here the implicit assumption is that on time scales of interest there are sufficient collisions to keep the loss cones filled, but not enough to invalidate the collisionless assumption for particles moving down the field line). Then the fraction of particles $\varepsilon_{L}$ which carries thermal energy out of the plasmasphere and into the ionosphere is given by the ratio of the loss cone solid angle $\Omega_{L}$ to the solid angle of the down going flux, $2 \pi$. From integration of angles over the loss cone, we obtain $\Omega_{L}=$ $2 \pi\left(1-\cos \alpha_{L}\right)$ and $\varepsilon_{L}=1-\cos \alpha_{L}$. From the invariance of the magnetic moment, we can define the loss cone at the position $(r, \lambda)$ by

$$
\frac{\sin ^{2} \alpha_{L}(r, \lambda)}{B(r, \lambda)}=\frac{1}{B_{L}\left(r_{L}, \lambda_{L}\right)}
$$

where $r$ is geocentric distance and $\lambda$ is magnetic latitude. With this implicit definition of $\alpha_{L}$, we can write the expression for $\varepsilon_{L}$, as

$$
\varepsilon_{L}(r, \lambda)=1-\sqrt{1-\frac{B(r, \lambda)}{B_{L}}}
$$

The modified thermal conductivity to be used in the simulations is then given by $\kappa_{c}=\varepsilon_{L} \kappa_{S H}$, where $\kappa_{S H}$ is the classical SpitzerHarm thermal conductivity [Spitzer and Harm, 1953].

By considering a dipole magnetic field, we can see that $B_{L}$ will vary somewhat with L-shell. But more variation for $\kappa_{c}$ will come from the fact that longer flux tubes with higher equatorial crossings will have a wider range of values for $B(r, \lambda)$. In Figure 2 , we plot values of $\varepsilon_{L}$ as a function of geocentric distance for different L-shells of a dipole field. From this figure, two aspects are readily apparent. First, the L-shell differences are relatively minor for a given altitude (providing the L-shell extends to the altitude of interest). Second, the modification factor decreases rapidly with increasing altitude. It will be noted that this factor varies continuously along the field line, being smallest at the highest altitudes (equatorial region), with a range of more than two orders of magnitude for $\mathrm{L}>2$.

\section{Comparisons of Simulations}

In order to determine the realistic consequences for plasma thermal structure of using the modified coefficient, we have carried out simulations with $\kappa_{c}$, which can be compared with similar simulations in which $\kappa_{S H}$ was used. The simulation code is the Field Line Interhemispheric Plasma (FLIP) program. This is a 1-D hydrodynamic code, containing full ionospheric chemistry and dynamics; it is described in detail by Richards et al. [1994]. To carry out the simulations, we have treated $\mathrm{H}^{+}$and $\mathrm{O}^{+}$as major ions and $\mathrm{He}^{+}$as a minor ion. For the simulations with $\kappa_{c}$, we compute $\varepsilon_{L}$ at each step in the calculation and apply it to $\kappa_{S H}$, as indicated above. For these simulations we took the loss altitude to be $500 \mathrm{~km}$.

Since the simulations of Comfort et al. [1995] motivated this study, we used the same conditions $(\mathrm{L}=3, \mathrm{~F} 10.7=231$, $\mathrm{LT}=$ $0730, \mathrm{Ap}=31$ ) so that we could compare results from this study with those simulations. In order to produce observed ion temperatures, using the classical thermal conductivity $\kappa_{S H}$ (as given by Banks and Kockarts, Chapter 22), the number $(\gamma)$ of heating units $\left(10^{9} \mathrm{eV} \cdot \mathrm{cm}^{-2} \cdot \mathrm{s}\right)$ required to be added directly to the ions was 8. In those simulations, this heat was distributed evenly over the flux tube above $3000 \mathrm{~km}$ altitude. (While no specific heating mechanism was invoked, it was suggested that Coulomb collisions with energetic particles or wave-particle interactions might cause such heating.) The ion temperature profile corresponding to this heat input is shown in Figure 3, labeled as $\kappa_{S H}, \gamma=8$. The other curves are for $\kappa_{c}$, with $\gamma$ taking on various values from 8 down to 1 . We see that simulated temperatures at the top of the flux tube are near those observed when $\gamma=8$ using the classical conductivity and when $\gamma=1.4$ using the modified thermal conductivity $\left(\kappa_{c}\right)$. Using $\gamma=8$ with $\kappa_{c}$ results in ion temperatures far larger than observed. With $\kappa_{c}$, considerably less heat is required to produce a given temperature, or conversely, a given amount of heating produces a much larger temperature increase at high altitudes.

To gain a clearer picture of these results, in Figure 4 we extract the profiles for $\gamma=1.4$ and add the corresponding electron temperature profiles. Note that the modified thermal conductivity has not been applied to the electrons, since our attention is focused on the ions. Comparing the two ion temperature profiles, we see that the profile for the modified conductivity case has lower temperatures at all altitudes between the top and the bottom, with smaller gradients near the bottom and larger gradients

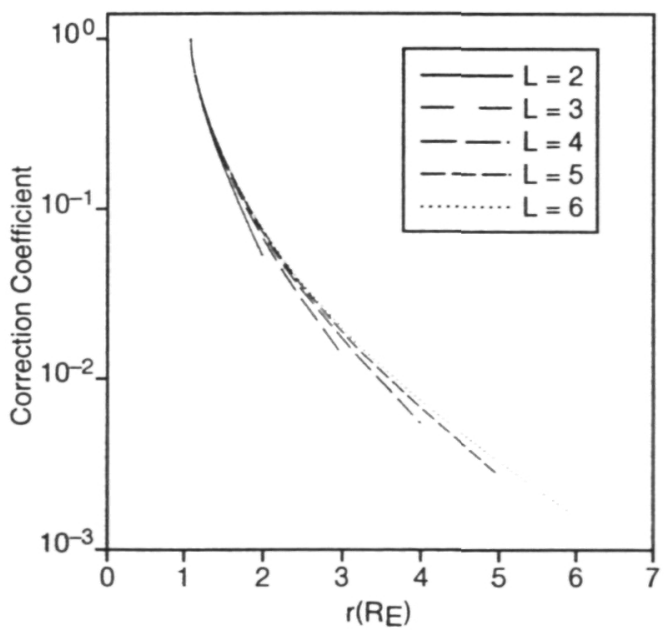

Figure 2. Thermal conduction reduction coefficient $\varepsilon_{L}$ as a function of geocentric altitude for L-shells 2 - 6 . 


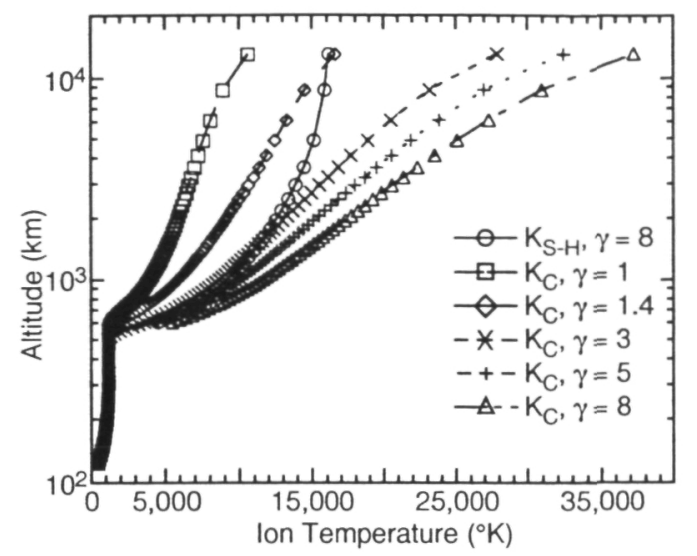

Figure 3. Simulated ion temperature profiles resulting from adding heat directly to ions for the case of the unmodified thermal conductivity $\kappa_{S H}$ and for the modified conductivity $\kappa_{c}$, with $\gamma$ as a parameter.

near the top. We can see that the correspondence between observed and simulated temperatures in the topside ionosphere is much closer with the modified conductivity, but the simulated temperature at lower altitudes $(\sim 800 \mathrm{~km})$ still remains somewhat large. The net consequence of all these factors is that less heat is conducted out of the plasmasphere to the topside ionosphere.

The other inconsistency between observations and simulation results which we were attempting to resolve was the effect the ion heating had on the plasma composition. Composition effects are seen in Figures $5 \mathrm{a}, \mathrm{b}$. The top panel displays the effects of the heating using the classical thermal conductivity, while the lower panel shows the effect of the modification. Clearly the most dramatic effects are at the top of the flux tube. $\mathrm{H}^{+}$densities are virtually unaffected, regardless of which $\kappa$ is used, and effects on $\mathrm{He}^{+}$are also small. However, $\mathrm{O}^{+}$densities have dropped significantly. Overall, heavy ion densities are still somewhat larger with $\kappa_{c}$ than observed, but much closer to the observations than for the unmodified case. This appears to be a consequence of the lower ion temperatures in the topside ionosphere where $\mathrm{O}^{+}$ is the dominant ion species.

\section{Discussion and Conclusions}

To resolve inconsistencies between simulated ion heating results and observations in low density flux tubes, we have proposed a mechanism which reduces the thermal coupling of the plasmasphere to the heat sink of the ionosphere. We suggest that

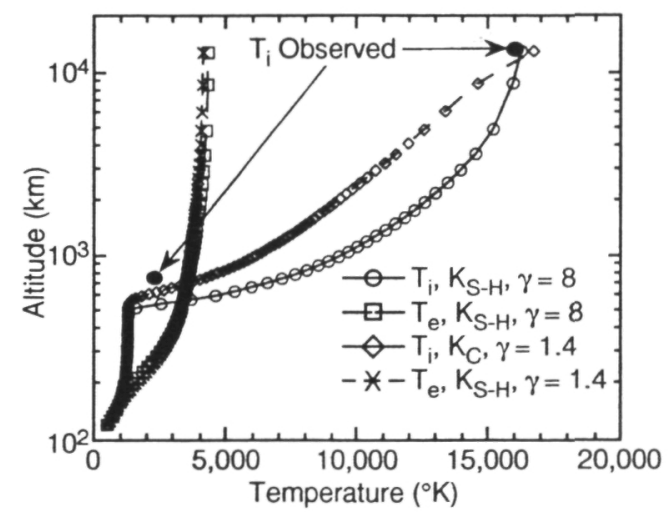

Figure 4. Simulated electron and ion temperature profiles for heating rates which provide ion temperatures close to those observed at high altitudes for cases with $(\gamma=1.4)$ and without $(\gamma=$ 8 ) modified ion thermal conductivity. High altitude observation is from DE1/RIMS and low altitude observation is from DE2/RPA [from Horwitz et al., 1990].
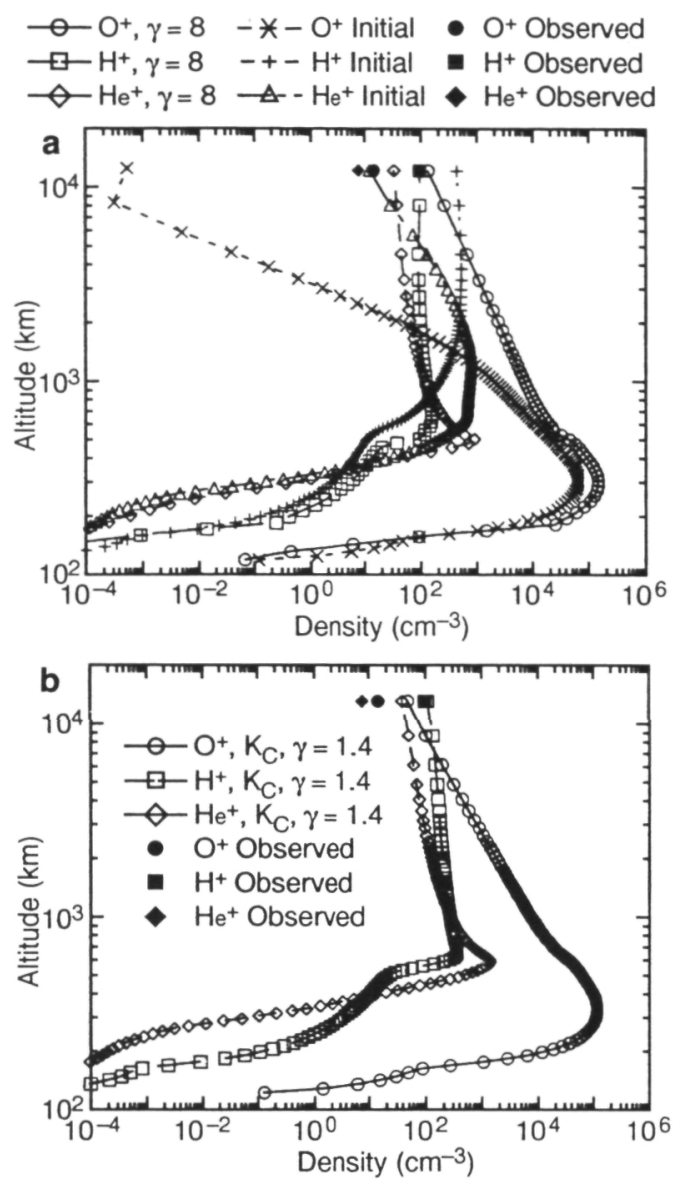

Figure 5. Composition results for the same simulations as in Figure 4; observations were made by DE1/RIMS. Panel $a$ shows the initial $\left((\gamma=0) \mathrm{H}^{+}, \mathrm{He}^{+}\right.$and $\mathrm{O}^{+}$density profiles and the final $(\gamma=8)$ density profiles for the unmodified ion thermal conductivity $\left(\kappa_{S H}\right)$. Panel $b$ shows the heated $(\gamma=1.4)$ density profiles for the modified case $\left(\kappa_{c}\right)$.

magnetic mirroring of quasi-collisionless particles, prevents the classical Spitzer-Harm thermal conductivity from providing a complete description of the heat flow for sufficiently low densities. One question which may be raised is how well the ions in our simulation satisfy the quasi-collisionless condition we postulate. To provide insight into this question, we have computed the mean time between collisions for protons with energies from 0.5 $\mathrm{eV}$ to $2.0 \mathrm{eV}$ for densities from $50 \mathrm{~cm}^{-3}$ to $500 \mathrm{~cm}^{-3}$, reasonable ranges for the outer plasmasphere; these are given in Table 1. We have also estimated transport times for protons with these same energies by considering the limiting case of particles moving along the field line for L-shells from 2 to 5 , from initial positions at the equator and at latitude $45^{\circ}$ down to the collision altitude $(500 \mathrm{~km})$; no forces are included in these simple estimates. Table 2 shows the resulting transport times. For the conditions of the simulation above $\left(\mathrm{n}=100 \mathrm{~cm}^{-3}, \mathrm{~L}=3\right)$, the ratio of the collision time to the transport time is about 2 for protons initially at the equator, which is in the appropriate range for the suggested model.

Table 1. Proton collision time (sec) as a function of energy and density

\begin{tabular}{clll}
\hline \multicolumn{4}{c}{ Energy $(\mathrm{eV})$} \\
\hline $\mathrm{n}\left(\mathrm{cm}^{-3}\right)$ & 0.5 & 1.0 & 2.0 \\
\hline 50 & 2178 & 6161 & 17430 \\
100 & 1089 & 3081 & 8713 \\
200 & 545 & 1540 & 4357 \\
500 & 218 & 616 & 1743 \\
\hline
\end{tabular}


Table 2. Proton transit time (sec) from initial point $(\mathrm{L}, \lambda)$ to $500 \mathrm{~km}$ altitude as a function of proton energy

\begin{tabular}{lllllll}
\multicolumn{8}{c}{ Energy $(\mathrm{eV})$} \\
\hline $\mathrm{L}$ & \multicolumn{2}{c}{0.5} & \multicolumn{2}{c}{1.0} & \multicolumn{2}{c}{2.0} \\
\hline$(\lambda)$ & $\left(0^{\circ}\right)$ & $\left(45^{\circ}\right)$ & $\left(0^{\circ}\right)$ & $\left(45^{\circ}\right)$ & $\left(0^{\circ}\right)$ & $\left(45^{\circ}\right)$ \\
\hline 2 & 1117 & $*$ & 790 & $*$ & 558 & $*$ \\
3 & 2029 & 354 & 1435 & 250 & 1015 & 177 \\
4 & 2934 & 700 & 2074 & 495 & 1467 & 350 \\
5 & 3836 & 1044 & 2712 & 738 & 1918 & 522 \\
\hline
\end{tabular}

This has basically been a 'proof of concept' investigation to determine whether or not the proposed mechanism would produce the type of effects the observations require. As such, it applies to physically limited conditions in which the densities are such that the plasma is in a quasi-collisionless to collisional regime. The simulations suggest that if the mechanism were operating, it would have the qualitative characteristics necessary to bring simulation results closer to observations. However, a more refined quantitative study (which we plan on carrying out) should consider additional aspects which are quantitatively important. For example, as seen in Figure 5 above, the number density varies along the field line so that the mean free path also varies with position. Similarly, the longer path lengths for the higher L-shells, make satisfaction of the collisionless criterion more restrictive for a given particle population. In addition, the particle path length is actually longer than the field line distance (guiding center path length), depending on the particle's equatorial pitch angle; the field line length is a lower limit. These effects should be folded into a density dependent modification to the thermal conductivity. A density dependence was developed by Mayr and Volland [1967] for electrons, but it had no relation to the effect of mirroring described in this report.

Since the Coulomb cross section is energy dependent, particles with different energies will have different mean free paths, as indicated in Table 1. For the quasi-Maxwellian velocity distributions expected for these conditions, it is possible that the high energy part of the distribution could satisfy the collisionless criterion, while the low energy part would not. A kinetic treatment will be required to examine this aspect in detail.

It appears likely that the mirroring mechanism proposed here can play a significant role in reducing the thermal coupling of the plasmasphere to the ionospheric heat sink, for the restricted conditions indicated. While the simple model used to test the idea is applicable under only these conditions, the physical decoupling of the ionosphere from the plasmasphere for particles outside the loss cone, due to magnetic mirroring, will occur for a broader range of lower densities. This mechanism is basically a kinetic phenomenon, and is beyond the means of even higher order hydrodynamic models to include. In this respect, the simple model suggested can be considered an attempt to extend the range of the hydrodynamic models into the quasi-collisionless regime with a physically-based, ad hoc modification. More detailed examination, with the aid of case-study observations and a kinetic simulation code, are needed to adequately determine the appropriate manner in which to employ this mechanism in a hydrodynamic code.

Acknowledgments. The work of RHC was partially supported by NASA Grants NAGW-1630 and NAG8-239, while that of PGR was partially supported by NASA Grant NAGW 996.

\section{References}

Banks, P. M., and G. Kockarts, Aeronomy, 2 vol., Academic Press, London, 1973.

Chapman, S. and T. G. Cowling, The Mathematical Theory of NonUniform Gases, 3rd ed., Cambridge University Press, London, Great Britain, 1970.

Comfort, R. H., I. T. Newberry, and C. R. Chappell, Preliminary statistical survey of plasmaspheric ion properties from observations by DE-1/RIMS, Modeling Magnetospheric Plasma, ed. T. E. Moore and J. H. Waite, Jr., Amer. Geophys. Un., 107-114, 1988.

Comfort, R. H., Richards, P. G., P. D. Craven, and M. O. Chandler, Problems in simulating ion temperatures in low density flux tubes, AGU Monograph on Coupling of Micro- and Mesoscale Processes in Space Plasma Transport, in press, 1995.

Craven, P. D., R. H. Comfort, P. G. Richards, and J. Grebowsky, Comparisons of modeled $\mathrm{N}^{+}, \mathrm{O}^{+}, \mathrm{H}^{+}$, and $\mathrm{He}^{+}$in the midlatitude ionosphere with mean densities and temperatures from Atmospheric Explorer, J. Geophys. Res., 100, 257-268, 1995.

Horwitz, J. L., R. H. Comfort, P. G. Richards, M. O. Chandler, C. R. Chappell, P. Anderson, W. B. Hanson, and L. H. Brace, Plasmasphere-ionosphere coupling II: ion composition measurements at plasmaspheric and ionospheric altitudes and comparison with modeling results, J. Geophys. Res., 95, 7949-7959, 1990.

Mayr, H. G., E. G. Fontheim, and K. K. Mahajan, Effect of modified thermal conductivity on the temperature distribution in the protonosphere, Ann. Geophys., 29, 21-24, 1973.

Mayr, H. G., and H. Volland, Model of magnetospheric temperature distribution, J. Geophys. Res., 73, 4851-4858, 1968.

Mitchell, H. G., Jr., S. B. Ganguli, and P. J. Palmadesso, Diodelike response of high-latitude plasma in magnetosphere-ionosphere coupling in the presence of field-aligned currents, J. Geophys. Res., 97, 12045-12056, 1992.

Richards, P. G., D. G. Torr, B. E. Reinisch, R. R. Gamache, and P. J. Wilkinson, F2 peak electron density at Millstone Hill and Hobart: Comparison of theory and measurement at solar maximum, J. Geophys. Res., 99, 15005-15016, 1994.

Spitzer, L., and R. Harm, Transport Phenomena in a completely ionized gas, Phys. Rev., 89, 977-981, 1953.

R. H. Comfort, Center for Space Plasma and Aeronomic Research, University of Alabama in Huntsville,Huntsville, AL 35899

P. D. Craven, Space Sciences Laboratory, NASA/Marshall Space Flight Center, Huntsville, AL 35816

P. G. Richards, Center for Space Plasma and Aeronomic Research, University of Alabama in Huntsville,Huntsville, AL 35899

(Received: April 3, 1995; revised: June 20, 1995; accepted: July 18, 1995) 